\title{
Photoproduction of isolated photons, inclusively and with a jet, at HERA
}

\section{Oleg KUPRASH*, for the ZEUS collaboration}

Deutsches Elektronen-Synchrotron DESY, Notkestr. 18, 22607 Hamburg, Germany

E-mail: oleg.kuprash@desy.de

The photoproduction of isolated photons, both inclusive and together with a jet, has been measured with the ZEUS detector at HERA using an integrated luminosity of $374 \mathrm{pb}^{-1}$. Differential cross sections are presented in the isolated-photon transverse-energy and pseudorapidity ranges $6<E_{T}^{\gamma}<15 \mathrm{GeV}$ and $-0.7<\eta^{\gamma}<0.9$, and for jet transverse-energy and pseudorapidity ranges $4<E_{T}^{\text {jet }}<35 \mathrm{GeV}$ and $-1.5<\eta^{\text {jet }}<1.8$, for exchanged-photon virtualities $Q^{2}<1 \mathrm{GeV}^{2}$. Differential cross sections as function of jet variables are also presented for direct and resolved photoproduction enhanced regions. Higher-order theoretical calculations are compared to the results.

XXII. International Workshop on Deep-Inelastic Scattering and Related Subjects 28 April - 2 May 2014

Warsaw, Poland

${ }^{*}$ Speaker. 


\section{Introduction}

Isolated photons produced in $\gamma^{*} p$ scattering are emitted by quarks and thus their measurement allows test both QCD and QED. Isolated photons are not affected by the hadronisation, therefore providing direct probe of the underlying partonic process. At HERA, electron-proton $e p$ collisions are dominated by the photoproduction (PHP) regime, where the virtuality of the exchanged photon is close to $Q^{2} \approx 0 \mathrm{GeV}^{2}$. Hence, distributions of the photon quantities can be measured more differentially compared to the isolated photon production in deep inelastic scattering (DIS).

In photoproduction, leading order (LO) QCD predicts two different mechanisms. In direct photoproduction the whole virtual photon participates in the hard subprocess, while in resolved photoproduction the photon fluctuates into a hadronic system prior to the interaction, and only part of the photon's energy is transferred to the struck parton of the proton. Resolved photoproduction is thus can be used to study the virtual photon structure. Both direct and resolved PHP are also sensitive to the proton structure. Examples of the LO diagrams for direct and resolved processes are shown in Figures 1(a) and 1(b), respectively.

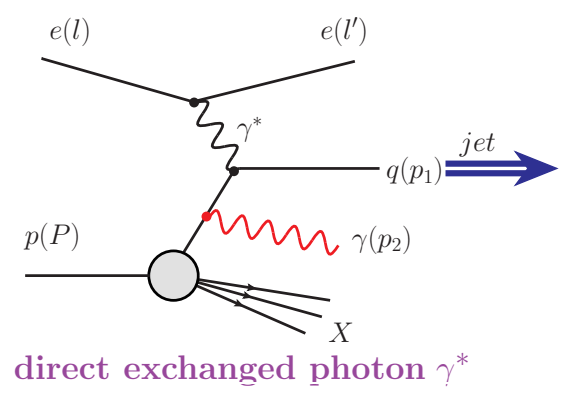

(a)

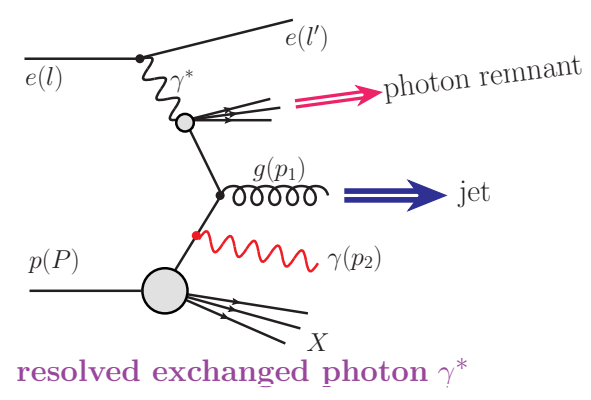

(b)

Figure 1: Contributions to the isolated photon production with direct (a) and resolved (b) virtual photon.

This report presents measurements of the isolated photon production [1, 2], with and without an accompanying jet, made by the ZEUS collaboration.

\section{Event Selection and Extraction of the Signal}

For the measurement, a data sample corresponding to an integrated luminosity of $374 \pm 7 \mathrm{pb}^{-1}$ was used. The sample contains both $e^{+} p$ and $e^{-} p$ data. The electron or positron beam energy was $E_{e}=27.5 \mathrm{GeV}$, while the proton beam energy was $E_{p}=920 \mathrm{GeV}$. The measurement was done with the ZEUS detector, detailed description of which can be found in [3]. The essential for this measurement part is the high-resolution uranium-scintillator calorimeter (CAL), consisted of three parts: the forward (FCAL), the barrel (BCAL) and the rear (RCAL). Each part of the CAL was subdivided into electromagnetic (EMC) and hadronic (HAC) cells. The electromagnetic calorimeter of the BCAL (BEMC) had a projecting geometry aimed at the nominal interaction point. The face dimensions of the BEMC cells were $5 \times 20 \mathrm{~cm}^{2}(5 \mathrm{~cm}$ along beam axis), providing a fine granularity allowing to separate isolated photons from the photon - products of neutral mesons decays (which are a main source of the background to the isolated photons). 
The photoproduction events are characterised by a very small recoiling angle of the scattered electron, which thus escapes undetected in the beam pipe. To select the PHP events, no scattering electron was allowed in the detector, and it was required $0.2<y_{\mathrm{JB}}<0.7$, where $y_{\mathrm{JB}}=$ $\sum_{i} E_{i}\left(1-\cos \theta_{i}\right) / 2 E_{e}$. Here $E_{i}$ and $\theta_{i}$ are the energy of the $i$-th calorimeter cell and its polar angle, respectively. The requirement on the position of a reconstructed primary vertex along the beam axis $Z,\left|Z_{\mathrm{vtx}}\right|<40 \mathrm{~cm}$, was imposed, to reduce the background from non-ep collisions.

The reconstruction of the photon candidate and the jets was done by analysing energy-flow objects (EFO), which are energy-momentum 4-vectors, constructed from clusters of calorimeter cells and, if available, associated tracks, reconstructed with the tracking detector.

The isolated photon candidates were reconstructed from EFO, with requirement that at least $90 \%$ of the reconstructed energy were measured in the BEMC and that there was no track associated with the EFO. It was required that a photon's candidate transverse energy, $E_{T}^{\gamma}$ was in the range $6<E_{T}^{\gamma}<15 \mathrm{GeV}$, and pseudorapidity, $\eta^{\gamma}$, in the range $-0.7<\eta^{\gamma}<0.9$, corresponding to the region covered by the BCAL.

The reconstruction of the jets was done using all EFOs of the event, with the $k_{T}$ clustering algorithm in the E-scheme in the longitudinally invariant inclusive mode with the radius parameter set to 1.0. Hence one of the jets was corresponding to, or containing the isolated photon candidate. It was required, that the ratio of the energies of the isolated photon candidate and the jet containing it, must be greater than 0.9 , to suppress contributions from the quark-to-photon fragmentation. When additional hadronic jet was required, its transverse energy, $E_{T}^{\text {jet }}$, was within the range $4<$ $E_{T}^{\text {jet }}<35 \mathrm{GeV}$ and the pseudorapidity, $\eta^{\text {jet }}$, within the range $-1.5<\eta^{\text {jet }}<1.8$.

Further isolation requirement was applied to reduce the background from the decay of neutral mesons within jets and from the quark-to-photon fragmentation: photon candidate must be isolated from tracks, demanding $\Delta R>0.2$, where $\Delta R=\sqrt{(\Delta \phi)^{2}+(\Delta \eta)^{2}}$ is the distance between the photon candidate and the nearest track with the momentum greater than $250 \mathrm{MeV}$.

As was mentioned above, photonic decays of the neutral mesons (mainly $\pi^{0} \rightarrow \gamma \gamma$ ) are the dominant background contribution to the sample of selected isolated photon candidates. However the width of an electromagnetic shower, produced in the BEMC by these background photons (always coming at least in pairs) is somewhat larger than those from the isolated photons. Therefore, the isolated photon signal can be extracted from the fit of the profile background and signal Monte Carlo (MC) histograms to the data. To generate the signal and background MC samples, the Pythia 6.416 [4] program was used. The MC samples were also produced Herwig 6.510 [5] program, to estimate systematic uncertainties. The generated with these programs events were passed through the full simulation of the ZEUS detector. Acquired information was used to correct the reconstructed with the detector information to a particle level (acceptance correction).

The discriminating variable to separate signal and background events, was chosen to be $\langle\delta Z\rangle=$ $\sum_{i} E_{i}\left|Z_{i}-Z_{\text {cluster }}\right| /\left(w_{\text {cell }} \sum_{i} E_{i}\right)$. This variable represents the mean energy-weighted width of the cluster of calorimeter cells, measured along the $Z$ axis. Here $Z_{i}$ is the $Z$ position of the centre of the $i$-th cell, $Z_{\text {cluster }}$ is the $Z$ position of the energy-weighted centre of the cluster, $E_{i}$ is the energy deposited in the $i$-th cell and $w_{\text {cell }}$ is the width of the cell in the $Z$ direction. The sum runs over all BEMC cells in the EFO, corresponding to the isolated photon candidate.

The distribution of the $\langle\delta Z\rangle$ variable is shown in Figure 2 for the data and for both signal and background $\mathrm{MC}$, for the inclusive isolated photon production. The distribution for the case 
of photon + jet production looks similar apart from lower by about $20 \%$ statistics. The signal was extracted by fitting $\langle\delta Z\rangle$ distribution separately in each bin of the cross section, in the range $0.05<\langle\delta Z\rangle<0.8$, using the minimum $\chi^{2}$ method. Obtained $\chi^{2}$ values per 28 degree of freedom were typically 1.1 .

\section{ZEUS}

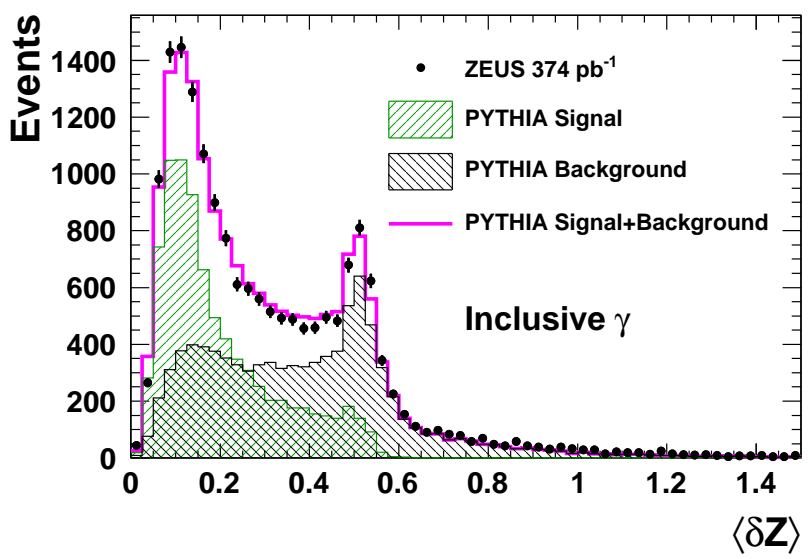

Figure 2: Distribution of $\delta Z$ for inclusive photon events, showing the fitted to the data signal and background MC components and their sum.

The following systematic uncertainties were taken into account:

- due to simulation of the hadronic final state: the cross sections were recalculated using the Herwig MC program. Resulting uncertainty was typically up to $8 \%$, rising in some regions of the phase space to $18 \%$;

- photon energy scale: the photon candidate energy was varied by $\pm 2 \%$ in the MC at the detector level, leading to $\approx 5 \%$ variation of the cross section;

- jet energy scale: if the jet was required, its energy was varied in the MC at the detector level, by amount decreasing from $\pm 4.5 \%$ to $\pm 2.5 \%$ depending on increasing $E_{T}^{\text {jet }}$. As a result, cross section varied by about $5 \%$;

- from variation of the relative fractions of the direct and resolved events in the total MC sample. leading to $\pm 2 \%$ change in the cross sections;

- upper limit of the $\langle\delta Z\rangle$ in the fit range was varied, to study the effect of the hadronic background modelling on the result. As a result, cross sections varied by $\pm 2 \%$.

Other sources of systematic uncertainty were ignored, after established to be negligible.

\section{Theoretical Predictions}

The measured cross sections were compared to two theoretical predictions. The next-toleading order (NLO) QCD predictions are provided by Fontannaz, Guillet and Heinrich (FGH) [6]. The proton and photon parton density functions (PDFs) used are CTEQ6 and AFG04, respectively.

The results were also compared to the $k_{T}$-factorisation approach, as provided by Lipatov, Malyshev and Zotov [7, 8, 9]. The unintegrated PDFs were used in the KMR formalism based on the MRST08 parton proton densities. 
Both theories include box contribution, which is formally one order in $\alpha_{s}$ (strong coupling constant) higher, but is known to be sizable. The uncertainties on the theoretical predictions in both cases are from the scale variations.

\section{Results}

The differential cross section of the inclusive isolated photon production as function of $E_{T}^{\gamma}$ is shown in Fig. 3(a). Both theories describe measured cross section very well within large theoretical uncertainties, indicating that inclusion of higher order correction would be desirable. Figure 3(b) shows the differential cross sections of the isolated photon with the accompanying jet production as function of $x_{\gamma}^{\text {meas }}$, which is defined as $x_{\gamma}^{\text {meas }}=\frac{E^{\gamma}+E^{\text {jet }}-p_{Z}^{\gamma}-p_{Z}^{\text {jet }}}{E^{\text {all }}-p_{Z}^{\text {all }}}$, where $E^{\text {all }}$ and $p_{Z}^{\text {all }}$ are obtained from the sum over all EFOs in the event. At LO, this variable gives a fraction of the virtual photon energy, transferred to a final state isolated photon and jet. In the $x_{\gamma}^{\text {meas }}$ region close to one, say $0.8<x_{\gamma}^{\text {meas }}<1$, almost full energy of the virtual photon is participating in the hard scattering, thus this region is dominated by the direct photoproduction. In contrast, lower $x_{\gamma}^{\text {meas }}$ region contains mostly resolved photoproduction events. The differential cross sections in $x_{\gamma}^{\text {meas }}$ are thus sensitive to the structure of the virtual photon. In the extended analysis of the isolated photon with jet production [2], differential cross sections in bins of ${ }_{\gamma}^{\text {meas }}$ were measured. As example, Figure 4 is showing cross sections as function of $E_{T}^{\mathrm{jet}}$ and $\eta^{\text {jet }}$ for $x_{\gamma}^{\text {meas }}<0.8$ (figures on top) and $x_{\gamma}^{\text {meas }}>$ 0.8 (bottom figures). The cross sections are typically well described by both models, except $\eta^{\text {jet }}$ at $x_{\gamma}^{\text {meas }}<0.8$ (top right) for the $k_{T}$ factorisation approach, which has different from measured shape. This may be connected with the procedure of setting the rapidity of the jet coming from the evolution cascade.

Generally, apart from the $\eta^{\text {jet }}$ at $x_{\gamma}^{\text {meas }}<0.8$, both theories describe measured cross sections well, however uncertainties of both theoretical predictions are much larger than those of the measured cross sections. Including higher order QCD corrections may be helpful to reduce the theoretical uncertainties.

\section{ZEUS}

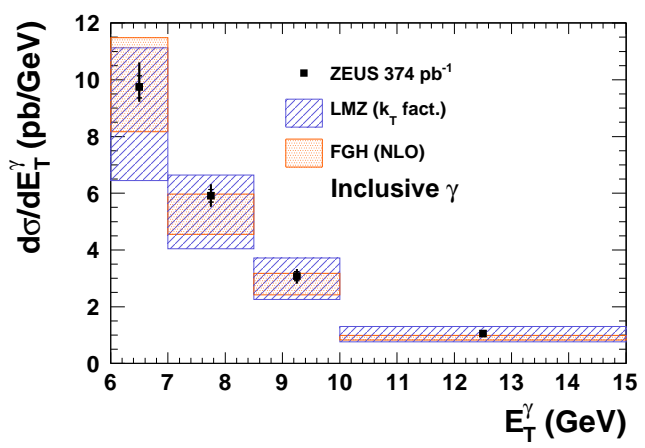

(a)

\section{ZEUS}

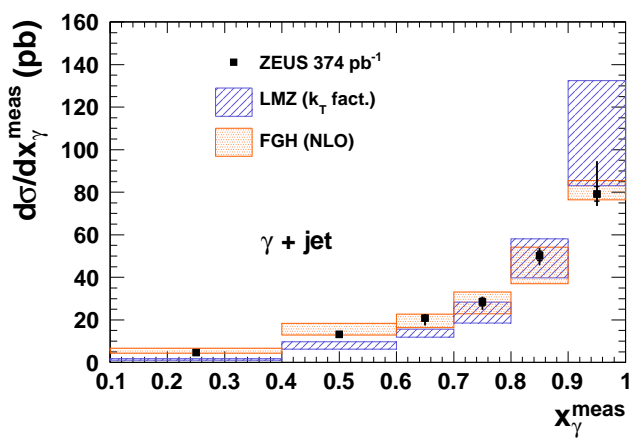

(b)

Figure 3: (a): Differential cross section of the inclusive isolated photon production as function of $E_{T}^{\gamma}$; (b): Differential cross section of the inclusive isolated photon + jet production as function of $x_{\gamma}^{\text {meas }}$. 


\section{ZEUS}


Figure 4: Differential cross sections as functions of $E_{T}^{\text {jet }}$ and $\eta^{\text {jet }}$ in the direct photoproduction enhanced $\left(x_{\gamma}^{\text {meas }}>0.8\right.$, top) and in the resolved photoproduction enhanced ( $x_{\gamma}^{\text {meas }}<0.8$, bottom) regions.

\section{References}

[1] H. Abramowicz et al. [ZEUS Collaboration], Phys. Lett. B 730 (2014) 293, DESY-13-234, arXiv:1312.1539 [hep-ex].

[2] H. Abramowicz et al. [ZEUS Collaboration], DESY-14-086, arXiv:1405.7127 [hep-ex].

[3] ZEUS Collaboration, U. Holm (ed.), The ZEUS Detector. Status Report (unpublished), DESY (1993), available on http://www-zeus.desy.de/bluebook/bluebook.html

[4] T. Sjostrand, S. Mrenna and P. Z. Skands, JHEP 0605 (2006) 026 [hep-ph/0603175].

[5] G. Corcella, I. G. Knowles, G. Marchesini, S. Moretti, K. Odagiri, P. Richardson, M. H. Seymour and B. R. Webber, JHEP 0101 (2001) 010 [hep-ph/0011363].

[6] M. Fontannaz, J. P. Guillet and G. Heinrich, Eur. Phys. J. C 21 (2001) 303 [hep-ph/0105121].

[7] A. V. Lipatov and N. P. Zotov, Phys. Rev. D 72 (2005) 054002 [hep-ph/0506044].

[8] A. V. Lipatov and N. P. Zotov, Phys. Rev. D 81 (2010) 094027 [arXiv:0907.3303 [hep-ph]].

[9] A. V. Lipatov, M. A. Malyshev and N. P. Zotov, Phys. Rev. D 88 (2013) 7, 074001 [arXiv:1307.3644]. 